\title{
Bee stings at sites of acupuncture as a potential therapy for idiopathic premature ovarian failure: a pilot study
}

\begin{abstract}
Objectives: To evaluate administration of bee stings at sites of acupuncture on the hormonal profile of patients with idiopathic premature ovarian failure (POF).

Study design: A pilot study carried at Obstetrics and Gynaecology Department, Tanta University Hospital, and at the Entomology Unit, Zoology Department, Faculty of Science, Tanta University from June 2015 to December 2015.

Patients and methods: 20 patients aged 18 to 40years with idiopathic POF were included in a case series study. Measurement of serum concentrations of Follicle Stimulating Hormone (FSH), Luteinizing Hormone (LH), 17 $\beta$-estradiol (E2) and Anti-Müllerian hormone (AMH) with calculation of Antral Follicles Count (AFC) and volume of ovaries was done before and after bee stings that were administered for 3 months, 2 sessions per week. Every session was 15 minutes with a range of 3-8 stings per session at the site of acupuncture. The outcome measures were changes in estimated variables within one month after bee stings.
\end{abstract}

Results: FHS and LH significantly decreased from $54.5 \pm 3.2$ to $27.1 \pm 5 \mathrm{mIU} / \mathrm{mL}$ and from $53.8 \pm 2.7$ to $25.2 \pm 4.1 \mathrm{mIU} / \mathrm{mL}$ respectively (P value $<0.001$ ). E2 significantly increased from $38.7 \pm 3.3$ to $92.5 \pm 2.9 \mathrm{Pg} / \mathrm{mL}$ ( $\mathrm{P}$ value $<0.05)$. Ovarian volume, $\mathrm{AFC}$ and $\mathrm{AMH}$ were not specifically different after bee stings.

Conclusion: Bee stings at the sites of acupunctures significantly reduced the serum levels of both FSH and LH and increased serum concentrations of E2 with improvement in hot flushes and vaginal dryness. Bee stings had no effect on AFC, AMH or ovarian volume. The use of bee venom (stings) therapy at the sites of acupunctures for patients with idiopathic POF is recommended.

Keywords: bee stings, acupuncture, premature ovarian failure
Volume 2 Issue 4 - 2016

\author{
Ayman Shehata,' Ayman Eldorf,' Naglaa \\ Hussien,' Nivin Naeem, ${ }^{2}$ Yahya Al Naggar, ${ }^{3}$ \\ John Giesy P4,5,6,7 \\ 'Department of Obstetrics and Gynecology, Tanta University, \\ Egypt \\ ${ }^{2}$ Department of Clinical Pathology, Tanta University, Egypt \\ ${ }^{3}$ Department of Zoology, Tanta University, Egypt \\ ${ }^{4}$ Department of Veterinary Biomedical Sciences and Toxicology \\ Centre, University of Saskatchewan, Canada \\ ${ }^{5}$ Department of Zoology and Center for Integrative Toxicology, \\ Michigan State University, USA \\ ${ }^{6}$ School of Biological Sciences, University of Hong Kong, Hong \\ Kong, China \\ ${ }^{7}$ State Key Laboratory of Pollution Control and Resource \\ Reuse, School of the Environment, Nanjing University, People's \\ Republic of China
}

Correspondence: Ayman Shehata, Obstetrics and Gynecology Department of Faculty of medicine, Tanta University, 31527 , Tanta, Egypt, Tel +201020972067,

Email ayman.dawood@med.tanta.edu.eg

Received: July 19, 2016 | Published: September 19, 2016

\section{Introduction}

Premature ovarian failure (POF) is an ovarian insufficiency in women under the age of 40years that is associated with serum concentrations of Follicle Stimulating Hormone (FSH) greater than $40 \mathrm{IU} / \mathrm{ml}$ with cessation of folliculogenesis and subsequently lesser production of estrogen. ${ }^{1}$ POF is characterized by amenorrhea and sometimes intermittent menstruation with unpredicted onset. Patients' presenting with POF, exhibit menopausal-like symptoms, such as hot flushes, dryness of the vagina and vaginit is with subsequent dyspareunia, mood changes and insomnia. ${ }^{1}$ POF affects approximately $1 \%$ of women under 40 years of age and $0.1 \%$ under 30 years. ${ }^{2}$ Symptoms of POF differ from those associated with menopause by the presence of varying unpredictable resumption of functioning of the ovaries, with successful pregnancy and delivery in approximately $5-10 \%$ of confirmed cases of POF. ${ }^{1,3}$ POF occurs due to idiopathic or genetic causes. Genetic causes include abnormalities of $\mathrm{X}$ chromosomes and single gene disorders. POF can also be associated with autoimmune diseases single or polyglandular immune disorder, viral infections, such as mumps, and oophoritis, and iatrogenic causes, such as chemotherapy and radiotherapy. Idiopathic or primary POF is a diagnosis of exclusion and constitutes about $75 \%-90 \%$ of all cases of POF. ${ }^{1,4}$
For diagnosis of POF, there must be at least two measurements in which menopausal levels of FSH (>40IU/L) are observed at least six weeks apart in a woman younger than 40years of age. In addition, elevated concentrations of Luteinizing Hormone (LH) and depressed concentrations of $17 \beta$-estradiol (E2) must also be observed. ${ }^{1}$ Additional laboratory investigations to elucidate possible causes of POF are requested upon clinical diagnosis of a specific disease or disorder. Trans-vaginal ultrasound examination of patients with POF shows diminished ovarian volume and numbers of Antral Follicles Count (AFC). Ovarian biopsy is not necessary for diagnosis of POF in patients with normal karyotype. ${ }^{5}$

Management of POF requires Hormonal Replacement Therapy (HRT) to relieve symptoms. HRT in these cases does not improve fertility, for which a range of medical treatments are used but the results are unsatisfactory. ${ }^{1}$ Use of Gonadotropin Releasing Hormone $(\mathrm{GnRH})$ analogues to suppress release of gonadotropin and resumption of the ovarian function has proved to be unsuccessful. ${ }^{6,7}$ Danazol, which is a derivative of the synthetic steroid ethisterone suppresses the production of gonadotropin and has some weak androgenic effects. However, Danazol, which in addition to its androgenic effect also has the side effect of modulating immune function, failed to reverse POF in patients with normal karyotype. ${ }^{8}$ Treatment with corticosteroids 
resulted in satisfactory ovulation and rates of pregnancy in cases of POF that were known to be caused by autoimmune diseases. ${ }^{9,10}$ Immunosuppressive agents were not effective and resulted in unwanted side effects. ${ }^{11}$ Clinical trials, in which treatment of POF by daily use of pentoxifylline combined with tocopherol and vitamin $\mathrm{E}$, for nine months or daily use of growth hormone-releasing, were ineffective for treatment of POF. ${ }^{12,13}$

Acupuncture has been used, in Asian countries for thousands of years, as a treatment for many disorders with few or no side effect. ${ }^{14}$ Acupuncture has been applied to a range of gynaecological disorders, including menopausal symptoms of normal women as well as patients subjected to oophorectomy. Acupuncture improves outcomes of pregnancy during in vitro fertilization (IVF). It is considered an effective and unexpansive treatment of polycystic ovary syndrome (PCO). The mechanism of action of acupuncture is not fully understood. Results of some studies have indicated acupuncture can modulate the hypothalamic-pituitary-ovary axis (HPOA). ${ }^{14-18}$

Administrations of bee venom through points of acupuncture (acupoint) was successful for producing a strong therapeutic effect, relative injection to non-acupoint areas ${ }^{19}$ and has been applied to treat several diseases, including Arthritis, Depression, Obsessive neurosis, Diabetic neuralgia, Sciatica and vitiligo. ${ }^{20}$ Bee venom contains 18 different compounds that all possess pharmaceutical properties. ${ }^{21-22}$ Apamin, melittin, phospholipase, and hyaluronidase in bee venom block or inhibit the nervous system, stimulate the heart and stimulate the adrenal glands. Also present in bee venom are antibiotics, phospholipase $\mathrm{A}$, as well as two amino acids rich in sulphur methionine and cystine. Sulphur is believed to be the main element in inducing the release of cortisol from the adrenal glands and in protecting the body from infections. Contact with bee venom produces a complex cascade of reactions in the human body. ${ }^{22}$ The aim of the current study was to evaluate administration effect of bee stings at the sites of acupuncture on hormonal profiles in patients with idiopathic POF.

\section{Patients and methods}

The current study is a consecutive case series prospective study which was conducted at the Obstetrics and Gynaecology Department, Tanta University Hospital, and at the Entomology Unit, Zoology Department, Faculty of Science, Tanta University from June 2015 to December 2015. The study was approved by the hospital ethical committee and patients signed an informed written consent after the reason for the study and methods to be applied were fully explained to them. The study included women were selected from those attending the Obstetrics and Gynaecology Department exhibiting idiopathic POF. The inclusion criteria were as follows:

i. Met POF diagnostic criteria, mainly with four months or longer of amenorrhea and serum concentrations of FSH greater than 40IU/L detected on at least two measurements at least one month apart

ii. Age was between 18 and 40; and

iii. Patients had stopped for at least three months prior to the study medications that can affect reproductive hormones.

Exclusion criteria were:

i. Patients subjected to bilateral oophorectomy or those who have chromosomal abnormalities or gonadal dysgenesis; ii. Had a history of receiving radiotherapy and/or chemotherapy for any type of cancer;

iii. Had a history of autoimmune disorders and/or of receiving hormonal treatments or been treated with immunosuppressant drugs;

iv. Exhibited a systemic, primary disease of liver, cardiovascular, kidney, or hematopoietic system; and

v. Had previous exposure to bee stings or any other type of apitherapy for any purpose within six months prior to the study and the presence or suspicion of allergy to bee stings. Patients were all subjected to examinations by trans-vaginal ultrasound and serum concentrations of hormones were also measured before the intervention.

Trans-vaginal ultrasound was done to exclude any ovarian organic pathology, to estimate the AFC and to calculate the ovarian volume according to the formula of prolate ellipsoid (Volume=length $\mathrm{x}$ height $\mathrm{x}$ width $\mathrm{x}$ 0.5233). ${ }^{23}$ Measurement of serum concentrations of FSH, LH, E2, and Anti-Müllerian hormone (AMH) was done for all patients prior to the intervention. Concentrations of AMH were quantified by use of an ELISA KIT (antibodies-online, catalogue number ABIN414951). It is competitive inhibition ELISA technique in which the limit of quantification is $37-3,000 \mathrm{pg} / \mathrm{ml}$ with intra-assay precision coefficient of variation $(\mathrm{CV})<15 \%$ and inter assay precision $\mathrm{CV}<15 \%$. Concentrations of LH were measured by use of an ELISA KIT (Cayman chemicals, item number 500720). It is a sandwich ELISA of which the limits of quantification are from 0.5 to $200 \mathrm{Iu} / \mathrm{ml}$ with an intra-assay variability $\mathrm{CV}<9.2 \%$ and inter- assay variability $\mathrm{CV}<7.9 \%$. Concentrations of FSH were measured by use of an ELISA KIT (Alpco, catalogue number 11-FSHHU-E01). This is a two-step capture ELISA technique with limit of quantification $5-100 \mathrm{Iu} / \mathrm{ml}$ with intra-assay variability $\mathrm{CV}<5.8 \%$ and inter-assay variability $<7.7 \%$. E2 was quantified using an ELISA KIT (R\&D systems, catalogue number KGE014) with lower and upper limits of quantifications 12.3-3000pg/ $\mathrm{ml}$ with intra-assay $\mathrm{CV}<8 \%$ and inter-assay $\mathrm{CV}<8.9 \%$.

\section{Intervention}

The intervention in the current study was administration of bee stings at the site of acupuncture. It is a pilot study and, to our knowledge, there are no previous published studies for use of bee sting in cases of POF. Hence, the sites of bee stings were selected to be the same sites used for acupunctures according to WHO Standardized Acupuncture Point Location. ${ }^{24}$ Sites used were ST 25 (Tianshu), ST 28 (Shuidao), ST 29 (Guilai), CV3 (Zhongji), CV 4 (Guanyuan), LR 3 (Taichong), GV 20 (Baihui), GV 24 (Shenting), GB 13 (Benshen), ST 36 (Zusanli), SP 6 (Sanyinjiao), KI 3 (Taixi), BL 23 (Shenshu) and BL 32 (Ciliao). GV 20 (Baihui), GV 24 (Shenting), GB 13 (Benshen), ST 36 (Zusanli), SP 6 (Sanyinjiao), and KI 3 (Taixi), BL 23 (Shenshu) and BL 32 (Ciliao).

Exposures to bee stings were conducted with worker honey bees (Apis mellifera $L$ ) of indeterminate age obtained from bee hives maintained in an apiary near the Agricultural Campus of Tanta University (Tanta, Egypt). Worker bees were brushed into disposable polyethylene bags then returned to the laboratory and placed in a refrigerator at $4^{\circ} \mathrm{C}$ for approximately $10 \mathrm{~min}$ to slow movement of bees. Bee stings were applied individually at sites of fertility acupuncture at Entomology unit. Duration of therapy was 3months with 2 sessions/week, every session was 15 minutes with a range of 
3-8 stings/session in order to cover all the above mentioned sites for all patients. Worker bees were forced to sting the back and abdomen at sites of acupuncture (Figures 1). After the bee's sting, the stinger was left in the body for about 15 minutes before it was removed. Within one month after intervention with stings of bees patients were subjected to another trans-vaginal ultrasound examination and blood drawn to quantify concentrations of hormones. The main outcomes of the study were changes in serum concentrations of the hormone, AFC and volume of ovaries after intervention with stings by bees.

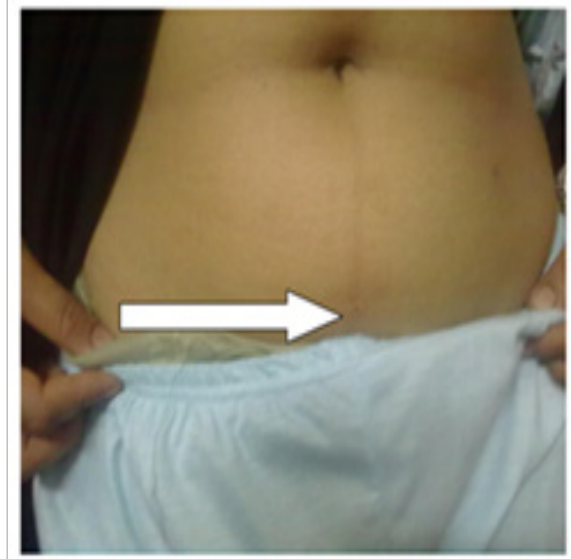

(A)

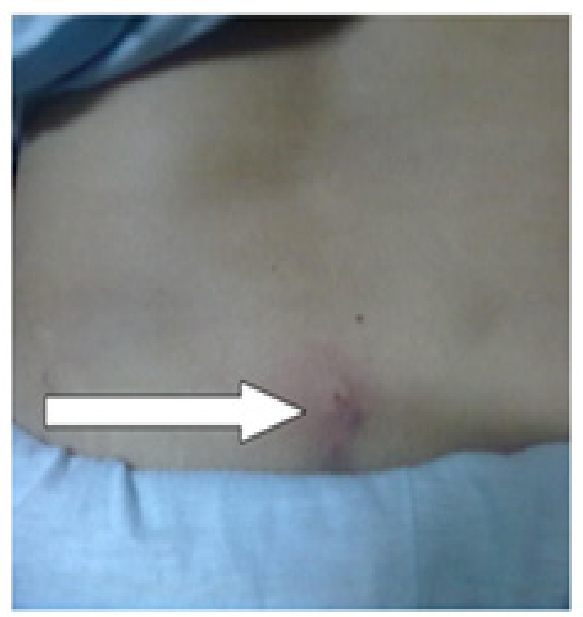

(B)

Figure I Bee sting sites (A) Anterior (B) Posterior.

\section{Statistical analyses}

Data were processed and analysed using statistical package for social science (SPSS) PC for windows XP (Version 16). Normality of data was assessed by use of the Kolomogrov-Smirnov test, and homogeneity of variance was determined with a Levene's test. If necessary to meet assumptions of parametric tests, data were $\log _{10}$ transformed to ensure normality and homogeneity of variance. Concentrations of hormones are presented as arithmetic mean and standard deviation, and for comparison between two groups, independent samples $(t)$ test was used with an alpha level of 0.05 .

\section{Results}

Twenty of 38 eligible patients were included in the final results, with 13 of those still in the follow-up beyond three months (Figure 2). Minimal allergic reactions were observed in four cases and were managed by oral antihistaminic then they completed the study. The demographic data of the 20 patients enrolled in the study are presented (Table 1).

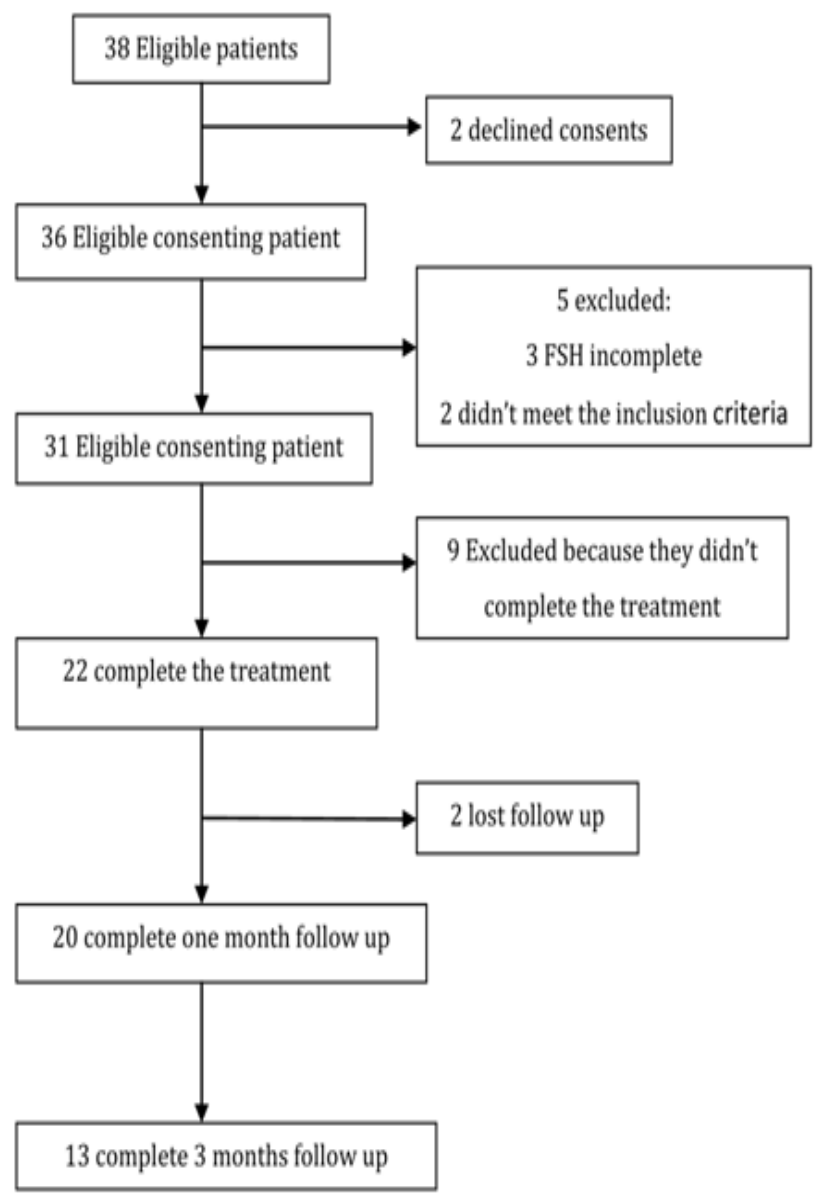

Figure 2 Selection of patients.

Table I Demographic data of patients enrolled in the study.

\begin{tabular}{lll}
\hline Parameter & Range & Mean \pm SD \\
\hline Age (years) & $18-33$ & $26.1 \pm 5.3$ \\
Age of menarche (years) & $12.8-16$ & $14.33 \pm 0.96$ \\
BMI (kg/M2) & $18.4-33.5$ & $26.74 \pm 5.87$ \\
Duration of Infertility (years) & $1.1-6.7$ & $4.1 \pm 1.91$ \\
\hline
\end{tabular}

Before the study amenorrhea was present in 14 patients (70\%) while Oligo-menorhea was found in six patients $(30 \%)$. Sixteen patients $(80 \%)$ were complaining of primary infertility and four patients $(20 \%)$ from secondary infertility. The other patients' complaints were hot flushes in eight patients (40\%), depression in 14patients $(70 \%)$, vaginal dryness in eight patients $(40 \%)$ and Libido disorders in two patients $(10 \%)$. Serum concentrations of the measured hormones, ovarian volume and AFC in the studied cases before and after bee sting are presented (Table 2). After bee sting therapy, there was a significant decrease in serum concentrations of both FSH and LH, while the two markers of ovarian reserve, $\mathrm{AMH}$ and $\mathrm{AFC}$ together 
with volume of the ovaries were not significantly affected (Table 2). After bee sting therapy, two patients regained menstruation; the first was after one month and the second after 3 months. After normalization of concentrations of $\mathrm{FSH}$ and $\mathrm{LH}$ to $6.3 \mathrm{IU} / \mathrm{ML}$ 7.6MIU/ML, respectively, pregnancy occurred in another patient who was 25 years old with secondary amenorrhea. This pregnancy was achieved after clomiphene citrate 50mg daily for five days starting in the second day of induced menstruation. Subjective improvement of vaginal dryness was observed in nine patients and severity of hot flushes was observed in seven patients.

Table 2 Selection of pateients

\begin{tabular}{llll}
\hline Parameter & $\begin{array}{l}\text { Before Bee } \\
\text { Stings }\end{array}$ & $\begin{array}{l}\text { After Bee } \\
\text { Stings }\end{array}$ & P-value \\
\hline FSH $(\mathrm{mlU} / \mathrm{mL})$ & $54.5 \pm 3.2$ & $27.1 \pm 5$ & $<0.001$ \\
$\mathrm{LH}(\mathrm{mlU} / \mathrm{mL})$ & $53.8 \pm 2.7$ & $25.2 \pm 4.1$ & $<0.001$ \\
Estradiol E2 $(\mathrm{Pg} / \mathrm{mL})$ & $29.5 \pm 2.9$ & $38.7 \pm 3.3$ & $<0.05$ \\
AFC & $2.8 \pm 0.3$ & $3.1 \pm 0.3$ & 0.166 \\
AMH $(\mathrm{Pg} / \mathrm{mL})$ & $0.51 \pm 0.6$ & $2.2 \pm 1.7$ & 0.213 \\
Ovarian volume $(\mathrm{mL})$ & $4.2 \pm 0.4$ & $4.1 \pm 0.3$ & 0.417 \\
\hline
\end{tabular}

\section{Discussion}

Results of the current study indicate that bee sting therapy at sites of acupuncture for patients with idiopathic POF can readjust their serum concentrations of FSH, LH and E2.These changes in concentrations of hormones were associated with partial improvement in symptoms, with resumption of menstruation in two cases with fewer incidences of hot flushes and decreased dryness of the vagina. These observed improvements in the symptoms can be explained by readjusted concentrations of hormones, especially those of E2. These readjustments of hormones were the cause for resumption of ultrasound documented ovulation in five patients after two to five cycles of induction. Moreover, one of these five patients achieved pregnancy after induced menstruation and induction of ovulation by use of clomiphene citrate. The relatively small rates of ovulation, which at the same time is considerable if compared with the results of other studies, ${ }^{6-8,11-13}$ can be attributed to low ovarian reserve of patients as showed by their lower AMH and AFC, before the study. Insufficient exposure to bee stings might also a cause of lesser rates of ovulation and pregnancy observed during the current study.

Although there is no standardized practice for administration of bee venom, some authors report that the location of the sting is important, with the sting acting as a sort of acupuncture in combination with the effects of the venom; while others report that the location is not important. Moreover, the number of stings also varies widely from a few to hundreds and they may be administered either by live bees or by injection. ${ }^{22}$ The use of bee stings at sites of acupunctures in the current study showed nearly similar results of acupunctures and electro-acupuncture in normalization of $\mathrm{FSH}, \mathrm{LH}$ and $\mathrm{E} 2$ in patients with idiopathic POF. In another study, acupunctures were used for 31 patients presenting with idiopathic POF at the same points used in the current study. In that study duration of acupuncture between 20-30minutes, once every other day for three months, ${ }^{25}$ the baseline serum concentrations of FSH and $\mathrm{LH}$ before acupuncture were $63.7 \pm 23.3$ and $48.6 \pm 18.7 \mathrm{IU} / \mathrm{L}$, respectively, after acupuncture, FSH significantly decreased to $48 \pm 16.6 \mathrm{IU} / \mathrm{L}(p=0.001)$ and LH significantly decreased to $17.01 \pm 11.66 \mathrm{IU} / \mathrm{L}(p=0.001)$. Serum concentration of E2 significantly increased from $49.5 \pm 30.5$ to $68.24 \pm 36.15 \mathrm{pmol} / \mathrm{L}$ $(p=0.001)$. In another study, electro-acupuncture (EA) was used in 21patients with idiopathic POF, EA stimulation in each patient was done for at least 20 minutes once daily and five times per week for the first four weeks, then once every other day and three times per week for the two months. ${ }^{26}$ They were then followed for three months. After one month, serum concentrations of FSH and LH decreased from $89.08 \pm 11.97$ and $37.1 \pm 3.47 \mathrm{IU} / \mathrm{L} 45.37 \pm 7.07$ and $22.08 \pm 3.66 \mathrm{IU} / \mathrm{L}$ respectively after the EA. After two months of EA, Concentrations of both of those hormones increased. Concentrations of FSH and LH were $49.28 \pm 8.85$ and $22.29 \pm 4.42 \mathrm{IU} / \mathrm{L}$, respectively, while concentrations of E2 increased from $33.35 \pm 18.83$ to $223.82 \pm 45.95 \mathrm{pmol} / \mathrm{L}$ after treatment by EA. Similar results to the current findings were observed by a study conducted in Japan. ${ }^{27}$ In that study menstruation in a highly trained athlete was restarted after using acupuncture with hormonal therapy, involving three times daily oral administration of $0.5 \mathrm{mg}$ norgestrel and $0.05 \mathrm{mg}$ ethinyl estradiol for two weeks. Using this hormonal combination with acupuncture was deemed to have given better results in regaining the menstrual blood flow and the biphasic basal body temperature than use of either therapy alone.

Effects of acupuncture on various gynaecological disorders are not fully explained and effects could be via various mechanisms. For instance, normalization of hormones after acupuncture might be due to modulation of the hypothalamic-pituitary-ovarian axis. ${ }^{25}$ Improvement in symptoms of patients was also related to improved mental state including rhythmic secretions of pituitary gonadotropin. Acupuncture can manage various gynaecological disorders, including POF through modulation of the autonomic nerve functions. ${ }^{26}$ Acupuncture in the lower abdomen enhances activities under control of the vagal innervation and suppress sympathetic activities simultaneously, this leads to stimulation of the autonomic innervation of the pelvic organs with increased their blood flow..$^{28}$ Acupuncture also has a central action that could explain its effect on different gynaecological diseases. Electro-acupuncture stimulates aromatization of androgen to estrogen at the hypothalamus ${ }^{29}$ and decreases the number of estrogen receptors at the hypothalamic preoptic area. ${ }^{30}$ More recently, by using fMRI acupuncture was found to modulate both electrical and enzymatic activities of the brain cortex.

In the current study bee stings had a similar modulatory effect on hormones as that obtained by acupuncture. This modulation of concentrations of hormones by bee venom might be due to any of the various components that have biological activities. At small doses of melittin, a biologically active peptide that constitutes about $50-55 \%$ of the total volume of bee venomis an anti-inflammatory agent. It increases capillary permeability and blood flow, interferes with coagulation of blood, modulates the immune system and affects the central nervous system. ${ }^{31-33}$ Phospholipase A and B in the bee venom interfere with the blood coagulation, improve blood flow, maintain integrity of neural tissues and act as de-toxicating agents. ${ }^{34}$ Adolapin is a peptide in venom of bees with anti-inflammatory and antirhumatic actions that inhibit cyclooxigenase and lipooxigenase enzymes in the brain and at the site stung. The authors hypothesized that components of bee venom have positive effects on the vascular and immune systems as well as on ovaries, that lead to re-adjusting its function together with improvement in central secretions in hormonal the brain..$^{34}$

In conclusion, Results of this study are unprecedented in 
suggesting that bee stings at sites of acupuncture significantly reduce concentrations of both FSH and LH in blood of patients with idiopathic POF. It also increases concentrations of E2 in blood serum, with partial improvement in hot flushes and dryness of the vagina. Alternatively, there were no statistically significant effects on AFC, AMH or volume of the ovaries. This effect of bee stings is exerted through the compounds present in bee venom which possess many biological activities. Results of this study recommend and support the use of bee venom (stings) therapy at the sites of acupunctures for patients with idiopathic premature ovarian failure. However, it is noteworthy that more detailed studies with a large number of patients and a standardized practice for the administration of bee venom are needed. Also, effect-directed fractionation and identification to determine which chemicals in venom of bees are responsible for the observed effects is needed.

\section{Acknowledgements}

None.

\section{Conflict of interest}

The author declares no conflict of interest.

\section{References}

1. Nelson LM. Clinical practice. Primary ovarian insufficieny. $N$ Engl $J$ Med. 2009;360(6):606-614.

2. Coulam CB, Adamson SC, Annegers JF. Incidence of premature ovarian failure. Obstet Gynecol. 1986;67(4):604-606.

3. Bakalov VK, Anasti JN, Calis KA, et al. Autoimmune oophoritis as a mechanism of follicular dysfunction in women with 46 , XX spontaneous premature ovarian failure. Fertil Steril. 2005;84(4):958-965.

4. Woad KJ, Watkins WJ, Prendergast D, et al. The genetic basis of premature ovarian failure. Aust N Z J Obstet Gynaecol. 2006;46(3):242-244.

5. Hoeck A, Schoemaker J, Drexhage HA. Premature ovarian failure and ovarian autoimmunity. Endocr Rev. 1997;18(1):107-134.

6. Ledger WL, Thomas EJ, Browning D, et al. Suppression of gonadotrophin secretion does not reverse premature ovarian failure. $\mathrm{Br} J$ Obstet Gynaecol. 1989;96(2):196-199.

7. Surrey ES, Cedars MI. The effect of gonadotropin suppression on the induction of ovulation in premature ovarian failure patients. Fertil Steril. 1989;52(1):36-41.

8. Anasti JN, Kimzey LM, Defensor RA, et al. A controlled study of danazol for the treatment of karyotypically normal spontaneous premature ovarian failure. Fertil Steril. 1994;62(4):726-730.

9. Cowchock FS, McCabe JL, Montgomery BB. Pregnancy after corticosteroid administration in premature ovarian failure (polyglandular endocrinopathy syndrome). Am J Obstet Gynecol. 1988;158(1):118-119.

10. Corenblum B, Rowe T, Taylor PJ. High-dose, short-term glucocorticoids for the treatment of infertility resulting from premature ovarian failure. Fertil Steril. 1993;59(5):988-991.

11. Kalantaridou SN, Braddock DT, Patronas NJ, et al. Treatment of autoimmune premature ovarian failure. Hum Reprod. 1999;14(7):1777-1782.

12. Letur-Konirsch H, Delanian S. Successful pregnancies after combined pentoxifylline-tocopherol treatment in women with premature ovarian failure who are resistant to hormone replacement therapy. Fertil Steril. 2003;79(2):439-441
13. Busacca M, Fusi FM, Brigante C, et al. Success in inducing ovulation in a case of premature ovarian failure using growth hormone-releasing hormone. Gynecol Endocrinol. 1996;10(4):277-279.

14. Qu F, Zhou J, Sang X, et al. Acupuncture and auricular acupressure in relieving menopausal hot flushes of bilaterally ovariectomized Chinese women: a randomized controlled trial. Evid Based Complement Alternat Med. 2011;713274.

15. Frisk J, Carlhäll S, Källström AC, et al. Long-term follow-up of acupuncture and hormone therapy on hot flushes in women with breast cancer: a prospective, randomized, controlled multicenter trial. Climacteric. 2008;11(2):166-174.

16. Kim DI, Jeong JC, Kim KH, et al. Acupuncture for hot flushes in perimenopausal and postmenopausal women: a randomised, sham-controlled trial. Acupunct Med. 2011;29(4):249-256.

17. Zheng CH, Zhang MM, Huang GY, et al. The role of acupuncture in assisted reproductive technology. Evid Based Complement Alternat Med. 2012;543924.

18. Lim DC, Chen W, Cheng LN, et al. Acupuncture for polycystic ovarian syndrome. Cochrane Database Syst Rev. 2011;(8):CD007689.

19. Kwon YB, Lee JD, Lee HJ, et al. Bee venom injection into an acupuncture point reduces arthritis associated edema and nociceptive responses. Pain. 2001;90(3):271-280.

20. Castro HJ, Mendez-Lnocencio JI, Omidvar B, et al. A phase I study of the safety of honeybee venom extract as a possible treatment for patients with progressive forms of multiple sclerosis. Allergy Asthma Proc. 2005;26(6):470-476.

21. Krell R. Value-added products from beekeeping. SAO Agricultural Services Bulletin. Rome: Food and Agriculture Organization of the United Nation; 1996.

22. Ali MA. Studies on bee venom and its medical uses. Int J Adv Res Technol. 2012;1(2):1-15.

23. Nwanko NC, Madufuro CO. Transvaginal Ultrasound measurement of ovarian volume in PortHarcourt. J Med Med Sci. 2011;2(9):1080-1084.

24. World health organization. WHO standard accupuncture point locations in the western pacific region. Philippines; 2008. p. 1-249.

25. Chen Y, Fang Y, Yang J, et al. Effect of acupuncture on premature ovarian failure: a pilot study. Evid Based Complement Alternat Med. $2014 ; 718675$

26. Zhou K, Jiang J, Wu J, et al. Electroacupuncture modulates reproductive hormone level in patients with primary ovarian insufficiency: results from a prospective observationl study. Evid Based Complement Alternat Med. 2013;657234.

27. Donoyama N, Hotoge S, Ohkoshi N. Acupuncture might have contributed to improving amenorrhoea in a top athlete. Acpunct Med. 2011;29(4):304-306.

28. Wang JD, Kuo TB, Yang CC. An alternative method to enhance vagal activities and suppress sympathetic activities in humans. Auton Neurosci. 2002;100(1-2):90-95.

29. Zhao H, Tian ZZ, Chen BY. Electroacupuncture stimulates hypothalamic aromatization. Brain Res. 2005;1037(1-2):164-170.

30. Ma S, Wu J, Feng Y, et al. Elevated estrogen receptor expression in hypothalamic preoptic area decreased by electroacupuncture in ovariectomized rats. Neurosci Lett. 2011;494(2):109-113.

31. Cho ZH, Hwang SC, Wong EK, et al. Neural substrates, experimental evidences and functional hypothesis of acupuncture mechanisms. Acta Neurol Scand. 2006;113(6):370-377. 
32. Bai L, Tian J, Zhong C, et al. Acupuncture modulates temporal neural responses in wide brain networks: evidence from fMRI study. Mol Pain. 2010;(6):73.

33. Son DJ, Lee JW, Lee YH, et al. Therapeutic application of anti-arthritis, pain-releasing, and anti-cancer effects of bee venom and its constituent compounds. Pharmacol Therapeutic. 2007;115(2):246-270.
34. Jeong JK, Moon MH, Bae BC, et al. Bee venom phospholipase A2 prevents prion peptide induced-cell death in neuronal cells. Int J Mol Med. 2011;28(5):867-873. 